\section{Análise da efetividade de um programa de incentivo ao aleitamento materno exclusivo em comunidade carente na cidade de São Paulo}

\section{Effectiveness analysis of an exclusive breast feeding program in a poor community in the city of São Paulo}

Nádia Zanon Narchi 1

Rosa Aurea Quintella Fernandes 2

Maria Magda Ferreira Gomes 3

Maria Luiza Queiroz 4

Daniela Novais Higasa 5

1 Faculdade de Enfermagem. Universidade Anhembi Morumbi. Rua Dr. Almeida Lima, 1134. Brás. São Paulo, SP, Brasil. CEP: 03.164-000

2 Mestrado em Enfermagem. Universidade Guarulhos, SP, Brasil ${ }^{3}$ Faculdade de Enfermagem e Nutrição. Universidade Federal de Mato Grosso, MT, Brasil

4,5 Curso de Graduação. Faculdade de Enfermagem. Santa Casa de São Paulo, SP, Brasil

\begin{abstract}
Objectives: to analyze effectiveness of an exclusive breast feeding program in a needy community in the city São Paulo in order to redirect its actions.

Methods: descriptive-exploratory survey, retrospective, quantitative with data collected in 56 medical files of women seen by the Program from August 2002 to November, 2003. Variables: employment status, age, number of children and type of care received during delivery and birth; adhesion and abandonment of medical visits; reasons for abandonment; nursing situation at discharge.

Results: $100 \%$ of the women were unemployed, $39.3 \%$ were adolescents, $48.2 \%$ were primiparous, $67.9 \%$ had normal delivery, $78.6 \%$ were roomed in with their babies and $68.6 \%$ did not refer to early contact with the baby following birth, 51.8\% adhered to the program and $48.2 \%$ abandoned the program for unknown reasons. When discharged, $17.3 \%$ of the mothers claimed to exclusive breast feeding for six months, $58.6 \%$ mixed and $24.1 \%$ used formulas. Early weaning occurred at an average of 96 days following birth.

Conclusions: the Program, notwithstanding an apparent low effectiveness needs to be continued because it benefits both mother and child especially in the social and economic conditions of that community although new strategies are required to improve adhesion and exclusive breast feeding averages.
\end{abstract}

Key words Breast feeding, Health promotion, Maternal-child nursing

\section{Resumo}

Objetivos: analisar a efetividade do Programa de Incentivo ao Aleitamento Materno Exclusivo implantado em uma comunidade carente do municipio de São Paulo a fim de redirecionar-lhe as ações.

Métodos: pesquisa descritivo-exploratória, retrospectiva, quantitativa, com dados coletados nas 56 fichas de mulheres atendidas pelo Programa no período de agosto de 2002 a novembro de 2003. Variáveis: situação empregatícia, idade, número de filhos e tipo de assistência recebida no parto e nascimento; adesão e abandono às consultas; motivos de abandono; situação do aleitamento no momento da alta.

Resultados: $100 \%$ das mulheres não trabalhavam, $39,3 \%$ eram adolescentes, 48,2\% eram primiparas, $67,9 \%$ referiram parto normal, $78,6 \%$ permaneceram em sistema alojamento conjunto e 68,6\% não referiram contato precoce com o bebê após o parto; $51,8 \%$ de adesão ao Programa e 48,2\% de abandono por motivos desconhecidos. No momento da alta, $17,3 \%$ das mães referiram aleitamento exclusivo durante os seis meses, $58,6 \%$ misto e $24,1 \%$ artificial. O desmame precoce ocorreu em média aos 96 dias de vida do bebê.

Conclusões: evidenciou-se que o Programa, apesar da aparente baixa efetividade, necessita ser mantido pelo beneficio que traz ao binômio mãe-filho tendo em vista as condições socioeconômicas daquela comunidade, sendo necessárias novas estratégias que melhorem a adesão e a média de aleitamento exclusivo.

Palavras-chave Aleitamento materno, Promoção da saúde, Enfermagem materno-infantil 


\section{Introdução}

$\mathrm{O}$ aleitamento materno é um ato universal e natural da mulher que propicia benefícios imensuráveis à criança, sendo por isso recomendado e estimulado por organismos mundiais ligados à saúde e ao bem-estar do menor. ${ }^{1} \mathrm{Na}$ verdade, por ser um ato natural, era de se esperar que todas as mulheres pudessem e quisessem amamentar seus filhos.

A amamentação ao seio materno deve começar tão cedo quanto possível, ser exclusiva e sob livre demanda até os seis meses de idade e ser mantida como complemento alimentar nos dois primeiros anos de idade da criança. ${ }^{1}$ No entanto, a literatura $2-18$ nos apresenta estatísticas desalentadoras, relacionadas à baixa prevalência do aleitamento materno, especialmente o exclusivo. Tal fato pode ser explicado tanto pela falta de conhecimento das mães sobre os benefícios, a importância do leite materno e a continuidade do aleitamento $2,3,7,12,14-16,18$ quanto pela indisponibilidade dos profissionais de saúde para ministrar orientações direcionadas à manutenção da amamentação ou, até, para manejar adequadamente a dieta infantil, ao orientarem precocemente o uso de chás, sucos e fórmulas lácteas. $4,5,12,17$

Frente a esta situação, o Ministério da Saúde tem incentivado a Iniciativa Hospital Amigo da Criança (IHAC), estratégia estabelecida, em 1992, pela Organização Mundial da Saúde (OMS) e o Fundo das Nações Unidas para a Infância (UNICEF) para estimular o aleitamento materno sendo adotada por serviços de saúde nas mais diversas regiões de nosso país. ${ }^{19}$ No entanto, a adesão a todos os dez passos dessa iniciativa avança com lentidão em muitos locais, dependendo da realidade local e institucional. Na cidade de São Paulo, por exemplo, no ano de 2001, mais de $1 / 4$ das maternidades públicas e 1/3 das maternidades privadas não cumpriam qualquer um dos dez passos, sendo que o cumprimento de pelo menos sete foi registrado em apenas dois hospitais públicos. ${ }^{20}$

Infelizmente, o que se percebe é que ainda ocorre descompromisso de muitos serviços e profissionais com a prática do aleitamento materno. Nesse sentido, não são raros os relatos de mães que referem, como causa do abandono do aleitamento exclusivo, o fato de na maternidade ter sido introduzida fórmula láctea como complemento da amamentação ou, mesmo, a falta de funcionários disponíveis para levar os recémnascidos até elas no horário das mamadas.

Além disso, observa-se a resistência, também de profissionais e instituições, nas abordagens de humanização da assistência ao nascimento e ao parto. Este fato tem influenciado negativamente o incremento da prática do aleitamento materno, haja vista, por exemplo, a dificuldade em implantar o primeiro passo para seu sucesso, que consite no contato precoce do binômio mãe-filho após o parto nas maternidades públicas e privadas do município de São Paulo. 20

Por outro lado, existem profissionais da área assistencial e acadêmica preocupados e empenhados com a expansão da prática do aleitamento materno na medida em que têm analisado sua situação em diferentes regiões do país, desenvolvido ações que possibilitam melhorar a adesão das mães ao aleitamento, mensurado e avaliado essas ações e divulgado os resultados de seus estudos.7,10-13,18,21,22

Diante desse panorama e considerando o aleitamento materno, principalmente o exclusivo, fundamental para a sobrevida e o desenvolvimento dos recém-nascidos, os pesquisadores do Núcleo São Lucas de Atendimento à Saúde da Mulher em São Paulo, decidiram investir seus esforços na temática, tendo em vista a taxa de apenas $28,7 \%$ de adesão referida por aquelas mães à amamentação, exclusiva ou não, nos seis primeiros meses. 21

Neste programa tem sido realizadas pesquisas sobre prevalência de amamentação22 e introdução de estratégias pedagógicas para orientação às mulheres na gestação e no pós-parto. 23,24

O objetivo deste estudo foi analisar a efetividade do referido Programa de Incentivo ao Aleitamento Materno Exclusivo a fim de redirecionar-lhe as ações, para que se possa obter maior adesão a ele.

\section{Métodos}

Trata-se de pesquisa descritivo-exploratória, retrospectiva, com abordagem quantitativa, desenvolvida com mulheres participantes do Núcleo São Lucas de Atendimento à Saúde da Mulher, inserido em uma comunidade carente do município de São Paulo. Salienta-se que todas as mulheres ao iniciarem a participação no Núcleo são informadas e consentem que os dados constantes de suas fichas sejam utilizados para trabalhos científicos, assinando o termo de consentimento livre e esclarecido. Além disso, este estudo foi aprovado pelo Comitê de Ética da Universidade Guarulhos, instituição a qual o projeto está vinculado.

O Núcleo São Lucas de Atendimento à Saúde da Mulher funciona em uma das creches da favela de Paraisópolis, comunidade com cerca de $60 \mathrm{mil} \mathrm{habi-}$ tantes, situada em área nobre da zona sul de São Paulo, SP, Brasil. A favela é servida por apenas uma unidade básica de saúde, que tem dificuldade em 
atender a demanda, especialmente na área maternoinfantil. Para a assistência ao parto não há referência garantida para as gestantes em nenhum hospital da região, ocorrendo a procura por vaga para internação obstétrica nas maternidades, todas com sistema alojamento conjunto implantado, mas nenhuma com o título de Hospital Amigo da Criança. Não há, também, nenhuma referência para o acompanhamento do binômio no pós-parto, que é complementado de forma restrita por algumas organizações sociais que atuam na comunidade, entre elas o Núcleo.

Em relação ao público alvo da área como um todo, dados do ano de 2001 mostram os números de 10.409 nascidos vivos e taxa de mortalidade infantil de 16,9 por 1.000 nascidos vivos. Quanto ao perfil da população atendida pelo Núcleo, os diversos trabalhos já realizados 21-24 permitem dizer que maior parte dessas mulheres vivem em situação de união não estável com seus parceiros, não trabalham, são jovens, provêm na sua maioria da região Norte-Nordeste, têm baixa escolaridade, vivem em moradias sem saneamento básico, têm em média dois a três filhos e apresentam dificuldade em gerir seu próprio sustento, pois referem situação permanente de desemprego familiar.

Os dados foram coletados nas fichas das 56 mulheres acompanhadas de agosto de 2002 a novembro de 2003, desde o primeiro retorno, após o parto, até a última consulta. Essas fichas contém dados referentes ao perfil socioeconômico e cultural, à história obstétrica pregressa e atual, à avaliação do aleitamento na primeira consulta e ao acompanhamento mensal do binômio.

Para a análise da efetividade do Programa de Incentivo ao Aleitamento Materno Exclusivo, foram analisadas nessas fichas todas as variáveis que se relacionavam direta ou indiretamente ao aleitamento, ou seja, os dados de caracterização das mulheres, como situação empregatícia, idade, número de filhos e tipo de assistência no parto e nascimento, a adesão e a taxa de abandono das mulheres às consultas no pós-parto, os motivos de abandono e, finalmente, a situação do aleitamento no momento da alta do Programa.

\section{Resultados}

As 56 mulheres acompanhadas tinham as seguintes características: $100 \%$ não trabalhavam, ou seja, não tinham nenhum registro de estabilidade empregatícia; 39,3\% eram adolescentes, na faixa etária entre 15 e 19 anos, 37,5\% tinham entre 20 e 25 anos e $23,2 \%$ idade superior a 26 anos; $48,2 \%$ estavam com seu primeiro bebê, $23,2 \%$ com o segundo e $28,6 \%$ com o terceiro, quarto ou quinto.
Quanto ao tipo de assistência ao parto e nascimento, $67,9 \%$ referiram parto normal e $32,1 \%$ cesárea; $78,6 \%$ afirmaram ter permanecido todo o tempo de internação hospitalar em sistema alojamento conjunto e $22,4 \%$ não desfrutaram desse tipo de acomodação, alegando problemas de saúde dos recém-nascidos; $31,4 \%$ referiram contato precoce com o bebê após o parto e $68,6 \%$ negaram esse contato.

Quanto à primeira consulta pós-parto no "Núcleo", essa ocorreu em média aos 40,2 dias de vida do bebê. Durante o acompanhamento dessas mulheres, $48,2 \%$ abandonaram o Programa, em média aos 71,3 dias de vida do bebê. Como houve abandono, des-conhecemse suas verdadeiras causas, no entanto, verificou-se que em todas as fichas havia a anotação de que algum outro tipo de leite já havia sido introduzido à dieta dos bebês ou que havia ocorrido o desmame.

As 51,8\% (29 mulheres) que foram acompanhadas durante os seis meses compareceram a uma média de 4,5 consultas. Em relação à situação do aleitamento no momento da alta do Programa, em média aos seis meses e meio de vida do bebê, 17,3\% delas referiram aleitamento exclusivo até o sexto mês, $58,6 \%$ misto e $24,1 \%$ artificial, constatando-se que o desmame precoce ocorreu em média aos 96 dias de vida do bebê.

\section{Discussão}

Os diversos trabalhos realizados pelo Núcleo São Lucas de Atendimento à Saúde da Mulher21-24 permitem descrever um perfil de população que apresenta diversas particularidades que interferem negativamente nas suas condições para o autocuidado, inclusive na manutenção do aleitamento materno exclusivo. Em relação à população deste estudo, os dados mostraram elevada frequência de mães adolescentes e primíparas, variáveis que se relacionam diretamente com a duração do aleitamento, pois mães com mais idade e maior número de filhos podem ter mais experiência ou maturidade para cuidar e amamentar. $14-16,25$

Nesse contexto, o oferecimento de informações sobre amamentação a essas jovens se justifica, especialmente quando são realizadas discussões em grupo e é fortalecida a autoconfiança no pós-parto, trabalho operacionalizado no Programa de Incentivo ao Aleitamento Materno Exclusivo do "Núcleo", que objetiva oferecer suporte para maior segurança das mães no que se refere ao cuidado do bebê e à iniciação e manutenção do aleitamento.

Os dados referentes à assistência durante o parto também podem ter interferido no processo de 
amamentação das mulheres deste estudo, pois existem evidências científicas de que a separação de mães e bebês após o nascimento exerce influência negativa sobre o sucesso do aleitamento. 25 Por outro lado, o contato precoce (Passo quatro da IHAC) favorece a prática do aleitamento materno, tanto imediatamente após o parto quanto aos dois a três meses mais tarde, recomendando-se o contato pele a pele dentro da primeira meia hora após o parto e continuado por pelo menos 30 minutos. Permite também que o bebê pegue o peito espontaneamente sempre que mostre sinais de que está preparado. 25

Esse contato normalmente é prejudicado quando ocorrem partos operatórios nos quais mãe e bebê são impossibilitados de interagir, tanto pelo posicionamento materno na cirurgia quanto pelas condições de saúde do recém-nascido. No entanto, mesmo quando mãe e bebê são separados por motivos justificáveis, pode haver sucesso no aleitamento, especialmente quando é dada orientação (Passo cinco da IHAC), ou seja, é oferecido apoio psicológico para desenvolver a auto-confiança da mãe e quando ela aprende como ordenhar o leite para alimentar seu bebê e manter a lactação. 25

Mesmo no caso de bebês prematuros ou com baixo peso ao nascer, fato ocorrido com apenas uma criança deste estudo, é possível obter sucesso na amamentação, dependendo para isso do apoio precoce e eficiente juntamente com a ordenha do leite. 25

Ainda assim, não havendo a possibilidade de contato precoce, do alojamento conjunto ser impedido por algum motivo, de serem utilizados suplementos durante o período de separação, é importante salientar que com um bom manejo do aleitamento materno, efeitos adversos podem ser superados 25 quando se conta com assistência adequada no pósparto, fato que tem sido observado nas consultas promovidas no "Núcleo" pois, às vezes, algumas mães voltaram a amamentar exclusivamente após as orientações recebidas durante as consultas.

A alta taxa inicial de adesão ao Programa e o pequeno número de faltas mostra o interesse de muitas mulheres em participar dele. No entanto, a elevada taxa de abandono demonstra a necessidade de melhorar a forma de acolher essas mulheres no pós-parto, de modo que elas possam ver utilidade nas consultas e não se sintam culpadas ou envergonhadas quando algo acontece, como, por exemplo, o desmame. Esta é uma evidência importante deste trabalho, ou seja, o grupo deve repensar a forma de abordar o binômio a fim de obter sucesso em seus propósitos.

Observou-se, ainda, que a maior parte das mães referiu aleitamento misto ou artificial aos seis meses de vida do bebê.

$\mathrm{O}$ acréscimo de algum outro tipo de alimento à dieta dos bebês ocorreu em média aos três meses de idade da criança, havendo grande parte das mulheres que referiram tê-lo feito por orientação de outros profissionais de saúde que realizam o acompanhamento dos bebês em serviços de puericultura, tanto da unidade básica de saúde da comunidade, quanto de outro serviço mantido por entidade filantrópica que atua junto às gestantes e seus bebês.

$\mathrm{O}$ índice de $82,7 \%$ de desmame precoce não foi muito diferente do encontrado em outros trabalhos $2,5,6,8,9,12,14-17$ os quais mostram acentuado declínio do aleitamento exclusivo, especialmente a partir do terceiro ou quarto mês de vida do bebê, e a alta taxa de desmame de bebês cujas mães não conseguem manter o aleitamento, mesmo não tendo a rotina de trabalho fora do lar, situação da população deste estudo.

É fato conhecido2,3,7-12,15,16,18 não ser tarefa fácil para a mulher amamentar e manter a amamentação. Os condicionantes sociais se sobrepõem aos biológicos e as mulheres sofrem as mais diversas pressões para introduzir novos alimentos. Talvez o caminho seja buscar formas não impositivas de mudar o olhar dessa mulher, de possibilitar-lhe conhecer e vivenciar de forma prazerosa o aleitamento. Além disso, o trabalho deve ser voltado à introjeção de um novo valor cultural em toda a sociedade. Isso implica em educação, suporte e harmonia de informações de todos que assistem e apoiam essa mulher no processo de aleitamento.

\section{Conclusões}

À primeira vista, os dados desta análise fazem com que se conclua pela baixa efetividade do Programa de Incentivo ao Aleitamento Materno Exclusivo. Também, as limitações relacionadas ao efeito dos pequeno número de observações e os viéses de seleção não permitem que se efetuem generalizações quanto às taxas de aleitamento exclusivo naquela comunidade carente. Mas esse não foi o objetivo deste estudo, pelo contrário, pretendeu-se verificar quais eram, ao final de um processo de acompanhamento, as taxas de sucesso do Programa e que correções de rumo deveriam ser realizadas a fim de que se obtivessem maiores percentuais de adesão ao aleitamento materno exclusivo.

Assim, conhecer esses dados e compará-los a outros de trabalhos semelhantes fez com os pesquisadores percebessem a necessidade de não só manter o "Programa" como também adotar novas 
estratégias a fím de melhorar a adesão e, especialmente, a média de aleitamento materno.

Desse modo, para o grupo de gestantes que iniciou o "Programa no Núcleo" após a coleta dos dados desse estudo, foram introduzidas medidas como acompanhamento de todos os bebês até o sexto mês, independentemente do tipo de aleitamento; busca ativa das mães faltosas por membros da própria comunidade, voluntários do "Núcleo", para conhecimento do motivo do abandono e tentativa de resgate ao Programa; agendamento da primeira consulta pós-parto o mais precocemente possível, ou seja, nos primeiros dez dias de vida do bebê; avaliação sistemática da técnica de aleitamento utilizada pelas mães, especialmente na primeira consulta; agendamento de retornos semanais ou quinzenais para os casos em que se percebam problemas na promoção e manutenção do aleitamento materno; introdução de atividades educativas na sala de espera que funcionem como grupos de apoio entre as mães; e, finalmente, elaboração de um programa que conte com a colaboração de membros da comunidade, para que eles sirvam de suporte às mulheres no processo de amamentação.

Essa última estratégia justifica-se em traba- lhos 18,25 que indicam que as práticas educativas sobre amamentação são mais úteis e eficazes quando há a participação de conselheiros leigos durante visitas domiciliárias. Este é o Passo 10 da IHAC, 25 que recomenda a utilização de um número de diferentes tipos de apoio pós-natal ao aleitamento como: aconselhamentos individualizados; ajuda centrada em dificuldades específicas ou em crises de auto-confiança da mãe; clínica de lactação; visitas domiciliárias; telefonemas; grupos de mães orientadoras e/ou grupos de apoio; envolvimento de familiares e amigos próximos e influentes na prática do aleitamento; e, por fim, grupos de apoio comunitários e de conselheiros.

É por isso que, além de todas as estratégias descritas, planeja-se convidar para participar dos grupos de apoio pessoas que convivem no ambiente social da mulher, tais como o pai, a avó da criança ou amigas íntimas, e que exercem influência na decisão de amamentar, especialmente das mães adolescentes, inseguras e inexperientes.

Finalmente, considera-se que investir no aleitamento materno, sem qualquer tipo de imposição, é uma tarefa social de relevância naquela comunidade, tendo em vista as circunstâncias em que as mulheres vivem e criam seus filhos.

\section{Referências}

1. OMS (Organização Mundial da Saúde). Proteção, promoção e apoio ao aleitamento materno: o papel especial dos serviços materno-infantis: declaração conjunta OMS/ UNICEF. Genebra; 1989.

2. Giugliani ERJ. O aleitamento materno na prática clínica. J Pediatr 2000; 76 [Supl 3]: 238-52.

3. Giugliani ERJ, Rocha VLL, Neves JM, Polanczyk CA, Seffrin CF, Susin LO. Conhecimentos maternos em amamentação e fatores associados. J Pediatr 1995; 71: 77-81.

4. César JÁ, Kuhn D, Devens ES, Martins Junior E, Aguiar MRC, Holthausen RS, Teixeira AMB, Horta BL. Prescrição de chás para crianças menores de seis meses: a opinião dos médicos de uma cidade de porte médio no Sul do Brasil. J Pediatr 1996; 72: 27-31.

5. Moura EFA. Duração do período de aleitamento materno de crianças atendidas em ambulatório de pediatria. J Pediatr 1997; 73: 106-10.

6. Vieira GO, Glisser M, Araújo SPT, Sales AN. Indicadores do aleitamento materno na cidade de Feira de Santana, Bahia. J Pediatr 1998; 74: 11-6.

7. Susin LRO, Giugliani ERJ, Kummer SC, Maciel M, Benjamim ACW, Machado DB, Barcaro M, Draghetti V. Uma estratégia simples que aumenta os conhecimentos das mães em aleitamento materno e melhora as taxas de amamentação. J Pediatr 1998; 74: 368-75.
8. Caldeira P, Goulart EMA. A situação do aleitamento materno em Montes Claros, Minas Gerais: estudo de uma amostra representativa. J Pediatr 2000; 76: 65-72.

9. Montrone VG, Arantes CIS. Prevalência do aleitamento materno na cidade de São Carlos, São Paulo. J Pediatr 2000; 76: 138-42.

10. Lamounier JÁ, Leão E. Estratégias para aumentar a prática da amamentação. J Pediatr 1998; 74: 355-6.

11. Figueiredo LMH, Goulart EMA. Análise da eficácia do programa de incentivo ao aleitamento materno em um bairro periférico de Belo Horizonte (Brasil): 1980/1986/1992. J Pediatr 1995; 71: 203-8.

12. Siqueira R, Durso N, Almada AGP, Moreira MT, Massad GB. Reflexões sobre as causas do desmame precoce observadas em dinâmicas de grupo de incentivo ao aleitamento materno. J Pediatr 1994; 70: 16-20.

13. Del Ciampo LA, Ricco RG, Muccillo G, Betiol H, Daneluzzi JC. Influências dos diferentes tipos de alojamento sobre recém-nascidos na prática do aleitamento materno. J Pediatr. 1994; 70: 10-5.

14. Lima TM, Osório MM. Perfil e fatores associados ao aleitamento materno em crianças menores de 25 meses na Região Nordeste do Brasil. Rev Bras Saúde Matern Infant 2003; 3 : $305-4$. 
15. Venancio SI, Escuder MML, Kitoko P. Frequência e determinantes do aleitamento materno em municípios do Estado de São Paulo. Rev Saúde Pública 2002; 36: 313-8.

16. Ramos CV, Almeida JAG. Aleitamento materno: como é vivenciado por mulheres assistidas em uma unidade de saúde de referência na atenção materno-infantil em Teresina, Piauí. Rev Bras Saúde Matern Infant 2003; 3: 315-21.

17. Pedroso GC, Puccini RF, Silva EMK, Silva NN, Alves MCGP. Prevalência de aleitamento materno e introdução precoce de suplementos alimentares em área urbana do Sudeste do Brasil, Embu, SP. Rev Bras Saúde Matern Infant 2004; 4: 45-58.

18. Montrone VG. Formação de agentes comunitários para a promoção do aleitamento materno e da estimulação do bebê. São Paulo: Manole; 2002.

19. Programa Nacional de Incentivo ao Aleitamento Materno. Grupo de Defesa da Saúde da Criança. Hospitais Amigos das Crianças: plano de ação. Brasília (DF); 1992.

20. Toma TS, Monteiro CA. Avaliação da promoção do aleitamento materno nas maternidades públicas e privadas do município de São Paulo. Rev Saúde Pública 2001; 35: 409-14.

21. Fernandes RAQ, Narchi NZ, Barreiros NF. Adesão das mulheres de uma comunidade carente ao aleitamento materno. In: II Encontro Internacional de Pesquisa em
Enfermagem; 2002; Águas de Lindóia, Brasil. [cd-room]. São Paulo: Escola de Enfermagem da Universidade de São Paulo; 2002.

22. Andreoni S, Fernandes RAQ, Narchi NZ. Programa de incentivo ao aleitamento materno em uma comunidade carente no município de São Paulo. In: III Congresso Brasileiro de Enfermagem Obstétrica; 2002 jul 18; Salvador, BA, Brasil. [cd-room]. Rio de Janeiro: Associação Brasileira de Obstetrizes e Enfermeiros Obstetras; 2004.

23. Narchi NZ; Fernandes RAQ; Barreiros NF. Educação em saúde e suporte familiar e social de gestantes de uma comunidade carente do município de São Paulo. In: III Congresso Brasileiro de Enfermagem Obstétrica; 2002 jul 18; Salvador, BA, Brasil. [cd-room]. Rio de Janeiro: Associação Brasileira de Obstetrizes e Enfermeiros Obstetras; 2004.

24. Fernandes RAQ. Estudo da morbimortalidade materna e perinatal e a qualidade da assistência pré-natal. Rev Paul Enferm 2001; 20: 57-67.

25. OMS (Organização Mundial da Saúde). Evidências científicas dos dez passos para o sucesso no aleitamento materno. Brasília (DF); 2001.

Recebido em 1 de março de 2004

Versão final em 10 de novembro de 2004

Aprovado em 29 de dezembro de 2004 